\title{
Steel Production Scheduling Based on Priced Timed Automata
}

\author{
Lixia $\mathrm{Ji}^{*}$ \\ Software Technology School, \\ Zhengzhou University, \\ Zhengzhou 450002, China \\ jilixia@zzu.edu.cn
}

\begin{abstract}
Scheduling production of steel is very complex because there are many different types of intricate constraints. This paper proposes a method using priced timed automata(PTA). PTA is the formal description method and it describes the behavior of entities, attributes and constraints. Furthermore, it adds some appropriate guidance to reduce the state space. This method converts the scheduling problem into state reachability analysis. Based on this method, we use price element to minimize the production cost.
\end{abstract}

Keywords-priced timed automata; steel production, cost-optimal; UPPAAL CORA.

\section{INTRODUCTION}

In recent years, the automation and security demand in computer control system for complex metallurgical process industry are increasing. Such complex systems are usually constructed of some digital components. Securities of the systems are strongly dependent on the interaction between the digital components in a sequential environment. This is the process controller and control.

\section{DESCRIPTION AND ANALYSIS}

The main steel production process: mining $\rightarrow$ beneficiation $\rightarrow$ sinter / pellet $\rightarrow$ puddling $\rightarrow$ steel $\rightarrow$ hot rolling $\rightarrow$ cold rolling $\rightarrow$ silicon steel[1,2]. The most important aspects is the steel smelting process, it starts from smelting furnace, then through converters and other equipments, and finally to casting machine. After this smelting process, raw materials become different quality steel and this process is shown in Fig. 1. In this process, the conversion of raw materials using a number of exclusive atomic operations. The processing and time of operation depend on the quality of the steel.

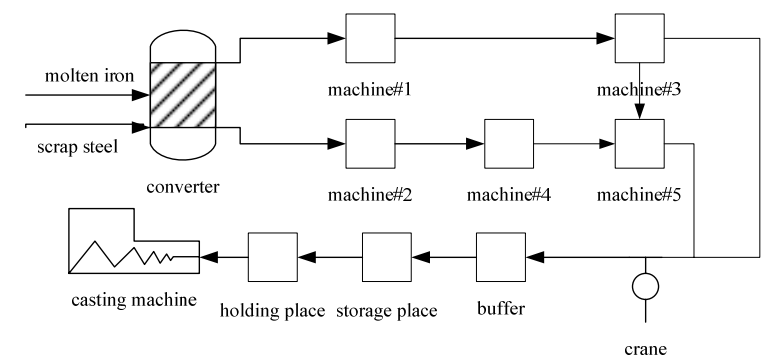

Fig.1. Diagram of steel smelting process
At first sight the problem looks like to a job-shop scheduling problem. The job-shop scheduling follows the following provisions. There are a number of machines and jobs. A job is defined as a sequence of operations which have to be executed in a given order. And each of these operations must be performed by a particular machine in a given period of time. In addition, studies often assume that machines are not allowed to be idle between two operations and each job can perform on each machine only once in an operation. Under this premise, job-shop scheduling problem is to schedule the operations in a way, which can minimize the time it takes to complete all jobs. On the basis of previous studies, there are many fast and efficient search algorithms to solve the job-shop scheduling problem. In steel production, there are no such assumptions. Worse, there are operations on certain machines that affect operations of other jobs on other machines. And operations that do not have a fixed operating time, jobs can't wait indefinitely for a machine to become free. It can even be difficult to decide whether there is a feasible schedule.

Timed automata has proven to be an effective method for formal modeling and verification of real-time systems. It can simulate the real-time requirements of the system. Priced timed automata method is a formal tool, it is expanded energy and time consumption costs based on timed automata method. PTA can take into account prices and costs when simulate the real-time systems. This paper describes the entities of steel production by the priced timed automata model, as well as the behavior, property and time constraints.

\section{COMPLEX PROCESS CONSTRAINTS}

\section{A. Recipe constraints}

As shown in Fig. 1, the part of the plant we consider are consists of two converters, five processing machines, one storage place, one buffer, two tracks (including crane), and one casting machine. Molten iron and scrap steel enter the system at converter. Material undergoes different treatments and moving through the system depending on the quality which has to be produced. The steel leaves the system at the casting machine, after which the ladle is transported to the storage place. The machines are used to perform different types of processing, they are divided into three categories. Machine\#1 and machine\#2 are identical and they are recorded as machine category A. And so on, machine\#3 and machine\#4 are machine category B, 
machine\#5 is machine category $\mathrm{C}$. Each process has a time limit and a lower limit, the recipe gives an upper and lower bound.

\section{B. Timing constraints}

In addition to recipe constraints, there are other timing constraints.

(1) When the converter is used, it has exclusive and do not allow other ladles to use.

(2) A crane need some time to pick and drop a ladle, it also need some time to move the ladle from one place to another.

(3) Before a ladle is allowed to enter the casting machine, it has to wait for a certain time in the holding place.

(4) The duration of a ladle in continuous casting machine is controllable within known bounds.

\section{Other constraints}

There is at most one ladle in one machine or between two machines, therefore, the machine and the position between two machines can be used as short-term buffer. The buffer place between the track and the storage place can hold at most five ladles. If there is already a ladle in the holding place, the ladle in continuous casting machine can only leave. As soon as a casting ladle has been completed, it can be taken away and start a new ladle.

\section{Cost constraint}

These constraints mentioned earlier, defines the security requirements for the plant. In order to reduce consumption costs, we have to look for a way it can have minimize production time based on meeting the objectives of each batch of product of quality.

\section{Priced Timed Automata Model}

\section{A. Using guided method appropriately}

Timed automata are widely used in various types complex real-time systems contain some trigger events and time constraints and it has been widely used in scheduling control and model checking[3]. Timed automata model can be used to describe any possible behaviors in the steel plant. In order to keep the size of the state space, we need to limit their behavior. We will do this by guiding the state space exploration according to a number of certain chosen strategies. The guided method are actualized in the model by introducing some new variables, invariants, constraints, and assignments to the new variables. In guiding mode, we reduce the irrelevant behaviors of the system and ensure that the timing model is valid. Overview of methodology in this article is shown in Fig. 2.

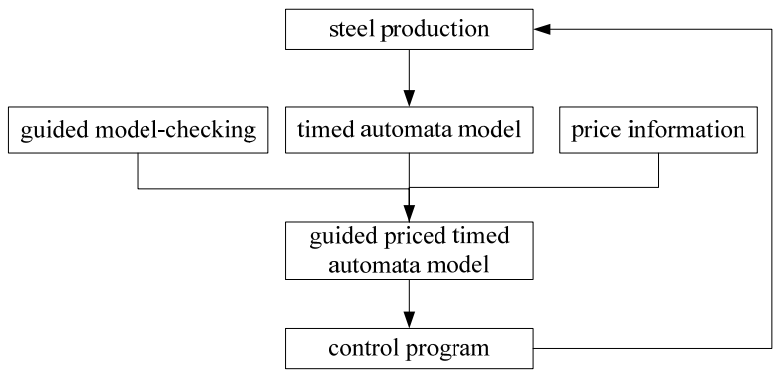

Fig.2. Overview of methodology

\section{B. Priced Timed Automata network}

Priced timed automaton[4] is an extension of timed automaton, it is a model that contains elements of the price. It is a formal method for modeling and solving the problem of the cost-optimal.

Definition 1 (Priced timed automata) [5]. A priced timed automaton is a 9-tuple PTA $=\left(L, l_{0}, C, A, E, I, V, P\right.$, $T)$. Where $L$ is a finite set of locations, $l_{0} \in L$ is a set of initial locations, $C$ is a finite set of clocks, $A$ is a finite set of actions, $E \subseteq L \times \Phi(C) \times \mathrm{A} \times 2^{C} \times L$ is the set of edges, an edge is generally accompanied by guards, action and clocks which will be reset, $I: L \rightarrow \Phi(C)$ is invariant of location, $V$ is a finite set of variables, $P: L \cup E \rightarrow \mathrm{N}_{0}$ is used to distribute price to locations and edges, $T: L \times \mathrm{A} \rightarrow L$ is a conversion function.

Multiple concurrent PTA may constitute priced timed automata network, each PTA has its own state, all PTAs share clock variables and global data variables, and they synchronize and collaborate with each others through the actions of edges. A pattern is used to describe running status of all the priced time automata in PTA network.

Definition 2 (Priced timed automata network). Suppose there are $n$ priced timed automata $\mathrm{PTA}_{1}, \ldots$, PTAn , a network of them $\mathrm{PTAN} \equiv \mathrm{PTA}_{1}\left\|\mathrm{PTA}_{2} \cdots\right\| \mathrm{PTA}_{\mathrm{n}}$ is the product of all the priced timed automata, a pattern of PTAN is a 3-tuple $c=<l, r, v>$, where :

$$
\begin{aligned}
& l=<l_{1}, \cdots, l_{\mathrm{n}}>, \forall 1 \leq i \leq \mathrm{n}, l_{i} \in L_{i}, \\
& r: \bigcup_{i}^{n} C_{i} \rightarrow R_{\geq 0} \text { performs the clock variables assignment, } \\
& v: \bigcup_{i}^{n} V_{i} \rightarrow \text { Int performs the numerical variables }
\end{aligned}
$$
assignment.

\section{Guiding Priced Timed Automata Models}

We use priced timed automata network to model the steel production, various entities and controls are modeled by priced timed automata. The PTA are combined using UPPAAL CORA's operation of parallel composition with handshake synchronization. There are mainly machines, continuous casting machines, crane, various constraints in PTA network of steel production. Basically there is one location for each state of entities. For each product line track there is a binary array used to store which positions are occupied or not. The various constraints are the invariant of the locations or transition conditions of the edges in priced timed automata models. And 
synchronization between different PTA realized by actions of the edges. Price parameter in PTA is used to solve the cost-optimal problem.

Part of the batch timed automaton is shown in Fig. 3[6], there are locations ila, i2, i2a, ilaa, i2aa, $k 1$ and cldown, where ila, i2a, ilaa and i2aa have a variant $x<=$ bmove, $x$ is clock variable of timed automata, the worst case time for moving a batch between two places is given by the constant bmove. ilaa is a invariant location, and its event left? of edge to ensure that the transition occurs within bmove time units.

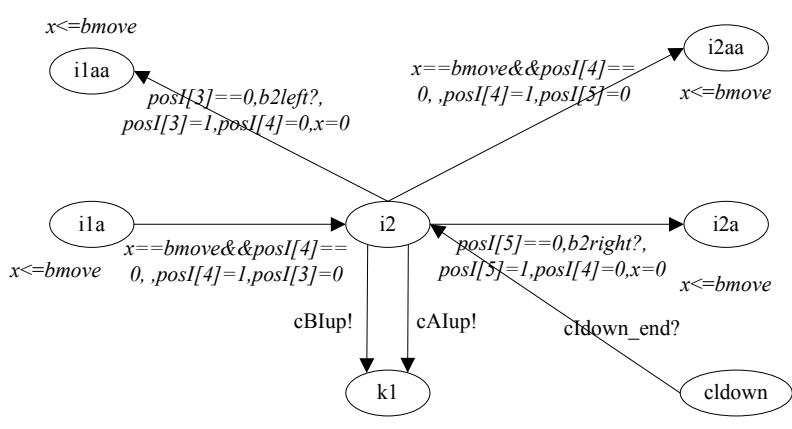

Fig.3. Part of the batch timed automaton

In timed automata description, the models allow any possible behavior. In this case, the state space explosion may occur. In order to reduce the state-space, we need appropriate restrictions in the model behavior. Here we do this by guiding the state-space exploration according to some certain chosen strategies. In this guidance, we have introduced some new variables and constraints. Of course the guidance is moderate, the goal is to reduce unnecessary behavior patterns, and we must also ensure that any guided model is an equally effective scheduling compared with the original model.

In the steel production process, if we consider only timing issues and the logical relationships between entities, price information is unimportant. Furthermore, if we also want to try to obtain optimal cost, price information will be considered. Here, we use a discrete variable price to represent the different branches may lead to cost changes. Guided part of the batch priced timed automaton according to Fig. 3 is shown in Fig. 4.

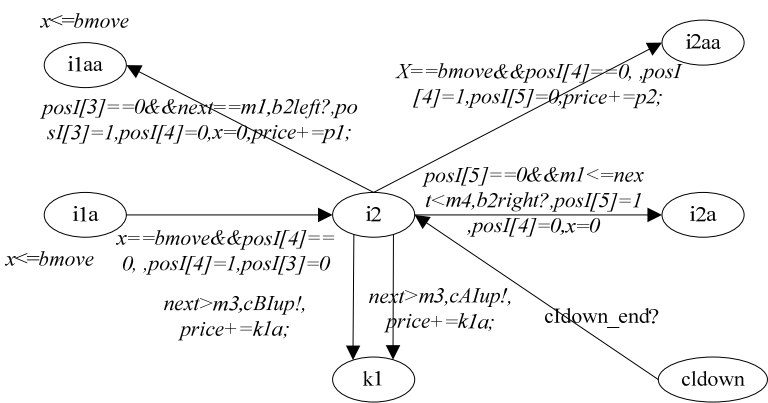

Fig.4. Guided part of the batch priced timed automaton

\section{COST-OPTIMAL REACHABILITY ANALYSIS}

Optimal cost reachability analysis is to find the minimum cost to reach a given target position, in the steel production is to find the lowest additional cost. Since the clock is defined as a nonnegative integer, the price conversion system can be infinite, costs and prices related to the status symbol may be infinite. In the exploration process, if the costs of along different paths to reach the same state are different, then it will discard the more expensive state. in the steel production PTA model, the cost is given by an affine plane over the clock zone[7].

In UPPAAL CORA, cost-optimal reachability analysis is performed using standard branch and bound algorithms defined as follows. The algorithm can use different search strategies, currently, are breadth-first, depth-first, best-first, random, best depth-first, and other user supplied heuristics. Costs: $=\infty$

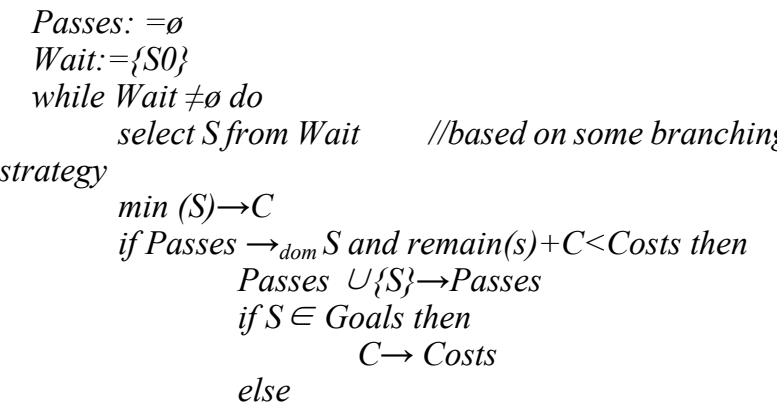

return Costs

$\left\{S^{\prime} \mid S^{\prime} \in\right.$ wait or $\left.S \rightarrow S^{\prime}\right\} \rightarrow$ Wait

The infinite variable Costs records the currently optimal known cost of reaching the goal location, lists Passes and Wait are used to recorded symbol status that have been explored or not. The algorithm iterates until no more states need to be explored. In the While loop, the algorithm chooses state $S$ based on the branch strategy. If $S$ is already explored or impossible to lower cost, then it will be skipped. Else if $S$ is the goal state we got a optimal cost, if not, we add all successors of S to Wait and continue to the next iteration.

\section{SIMULATION AND ANALYSIS}

In this section, we model ten batches of two different qualities steels. The quality 1 requires 1 time unit at machine category A and 1 time unit machine category B. The recipe for the quality 2 asks for treatments at machine category A, B and C, taking 1 time unit all. Filled ladles are not allowed to stay for more than four time units in the system. We used timed automata models and guided priced timed automata models respectively, both simulation experiments were carried out five times. The following table shows the simulation results.

As can be seen from Table 1, the simulations using guided priced timed automata are deadlock free. Under the premise of the same raw material, average time decreased $9.2 \%$, the average cost (excluding raw materials) decreased $9.9 \%$. There was not significant increase in steel product value. In short, this method can find a viable production 
sequence, avoiding the generation of deadlock, and the production costs have a significant reduction. Since the simulation does not take into account many confounding factors, the method still needs to research furthermore.

\section{SUMMARY}

According to a variety of different types of complex constraints in steel production scheduling, this paper presented a method, it modelled the production process using priced timed automata, and used appropriate guidance to avoid the state space explosion and deadlock problems. The method converted the scheduling problem into a reachability problem, and reduced the consumption costs of the production process. It provided a scientific advice to steel production scheduling.

\section{CORRESPONDING AUTHOR}

Lixia Ji, jilixia@zzu.edu.cn, 18539293066

\section{REFERENCES}

[1] Shi Yongtao, Study on Simulation Model of Workshop Scheduling for Transportation of Molten Iron in New Steel Manufacturing Process. College of Economics and Business Administration of Chongqing University, Chongqing, China( 2011)

[2] Ansgar Fehnker, Scheduling a Steel Plant with Timed Automata, rtcsa pp.280, Sixth International Conference on Real-Time Computing Systems and Applications (1999)

[3] ZHOU Qing-lei,JI Li-xia. Model checking of real-time systems based on UPPAAL. Computer Applications(2004)

[4] G. Behrmann, G, K.G. Larsen, J. I. Rasmussen. Priced timed automata: Algorithms and applications[C]. In International Symposium Formal Methods for Components and Objects (2004)

[5] JI Li-xia, ZHANG Han. Aircraft landing scheduling based on priced timed automata in complex.Transducer and Microsystem Technologies(2013)

[6] Thomas Hune , Kim G. Larsen,Paul Pettersson. Guided Synthesis of Control Programs Using UPPAAL.

[7] K. Larsen, G. Behrmann, E. Brinksma, et al. As cheap as possible: Efficient cost-optimal reachability for priced timed automata. In Proc. of Computer Aided Verification(2011, 2102)

TABLE 1. SIMULATION SCHEDULING RESULTS ANALYSIS

\begin{tabular}{|c|c|c|c|c|c|c|c|}
\hline Method & No. & $\begin{array}{c}\text { Deadlock } \\
\text { (T/F) }\end{array}$ & $\begin{array}{l}\text { Quality } 1 \\
\text { (ton) }\end{array}$ & $\begin{array}{l}\text { Quality } 2 \\
\text { (ton) }\end{array}$ & $\begin{array}{l}\text { Time-consuming } \\
\text { (clock time unit) }\end{array}$ & $\begin{array}{c}\text { Cost of } \\
\text { production } \\
\text { (Ten thousand } \\
\text { yuan) }\end{array}$ & $\begin{array}{c}\text { Product value } \\
\text { (Ten thousand } \\
\text { yuan) }\end{array}$ \\
\hline \multirow{4}{*}{ TA } & 1 & $F$ & 350 & 157 & 34 & 2.57 & 245 \\
\hline & 2 & $\mathrm{~T}$ & - & - & - & - & - \\
\hline & 3 & $\mathrm{~T}$ & - & - & - & - & - \\
\hline & 4 & $\mathrm{~F}$ & 352 & 224 & 31 & 2.48 & 253 \\
\hline Av. & & & & & 33.7 & 2.63 & 234 \\
\hline \multirow{5}{*}{ Guided PTA } & 1 & $\mathrm{~F}$ & 352 & 158 & 29 & 2.24 & 220 \\
\hline & 2 & $\mathrm{~F}$ & 290 & 282 & 31 & 2.56 & 229 \\
\hline & 3 & F & 405 & 168 & 30 & 2.25 & 246 \\
\hline & 4 & $\mathrm{~F}$ & 292 & 278 & 33 & 2.64 & 256 \\
\hline & 5 & $\mathrm{~F}$ & 333 & 245 & 30 & 2.17 & 256 \\
\hline Av. & & & & & 30.6 & 2.37 & 241 \\
\hline
\end{tabular}

